\title{
Fetal and neonatal exposure to trans-fatty acids impacts on susceptibility to atherosclerosis in apo $E^{*} 3$ Leiden mice
}

\author{
Louise Gates ${ }^{1}$, Simon C. Langley-Evans ${ }^{1}$, Jana Kraft ${ }^{2}$, Adam L. Lock ${ }^{3}$ and Andrew M. Salter ${ }^{1 *}$ \\ ${ }^{1}$ School of Biosciences, University of Nottingham, Sutton Bonington, Loughborough, LE12 5RD, UK \\ ${ }^{2}$ Department of Animal and Veterinary Sciences, University of Vermont, Burlington, VT 05405, USA \\ ${ }^{3}$ Department of Animal Science, Michigan State University, East Lansing, MI 48824-1225, USA \\ (Submitted 29 July 2016 - Final revision received 9 December 2016 - Accepted 5 January 2017 - First published online 22 February 2017)
}

\section{Abstract}

Nutrition during pregnancy can impact on the susceptibility of the offspring to CVD. Postnatal consumption of trans-fatty acids (TFA), associated with partially hydrogenated vegetable oil (PHVO), increases the risk of atherosclerosis, whereas evidence for those TFA associated with ruminant-derived dairy products and meat remain equivocal. In this study, we investigate the impact of maternal consumption of dietary PHVO (P) and ruminant milk fat (R) on the development of atherosclerosis in their offspring, using the transgenic apoE*3 Leiden mouse. Dams were fed either chow (C) or one of three high-fat diets: a diet reflecting the SFA content of a 'Western' diet (W) or one enriched with either $\mathrm{P}$ or R. Diets were fed during either pregnancy alone or pregnancy and lactation. Weaned offspring were then transferred to an atherogenic diet for 12 weeks. Atherosclerosis was assessed as lipid staining in cross-sections of the aorta. There was a significant effect of maternal diet during pregnancy on development of atherosclerosis $(P=0.013)$ in the offspring with those born of mothers fed $\mathrm{R}$ or $\mathrm{P}$ during pregnancy displaying smaller lesions that those fed $\mathrm{C}$ or $\mathrm{W}$. This was not associated with changes in total or lipoprotein cholesterol. Continuing to feed $\mathrm{P}$ during lactation increased atherosclerosis compared with that seen in offspring of dams fed $\mathrm{P}$ only during pregnancy $(P<0 \cdot 001)$. No such effect was seen in those from mothers fed $\mathrm{R}(P=0.596)$ or $\mathrm{W}(P=901)$. We conclude that dietary TFA have differing effects on cardiovascular risk at different stages of the lifecycle.

\section{Key words: Trans-fatty acids: Maternal diets: Programming: Atherosclerosis}

Both human epidemiological studies and animal experiments, show that maternal diet during pregnancy impact the susceptibility of the offspring to a range of chronic diseases. Poor maternal nutrition is frequently associated with low birth weight and subsequently increased susceptibility to hypertension, obesity, type 2 diabetes and atherosclerotic $\mathrm{CVD}^{(1-4)}$. Fetal exposure of rats to maternal low-protein diets results in hypertension $^{(5)}$, impairments in renal development ${ }^{(6)}$, and abnormalities in $\operatorname{lipid}^{(7)}$ and glucose homoeostasis ${ }^{(8)}$. In the ApoE*3 Leiden mouse we have demonstrated that maternal low-protein diets during pregnancy induced hypercholesterolaemia and increased atherosclerosis in the offspring ${ }^{(9)}$.

More recently, it has also become apparent that maternal overnutrition, particularly energy dense, high-fat diets, rich in SFA, often resulting in maternal adiposity, can also programme metabolic diseases in the offspring. Risk of CVD and premature death in the offspring of obese women has recently been demonstrated $^{(10)}$. In animal studies, maternal obesity is associated with increased obesity, impaired glucose homoeostasis and hypertension in the offspring ${ }^{(11)}$. Maternal hypercholesterolaemia is associated with increased susceptibility to atherosclerosis in both animal models ${ }^{(12)}$ and humans ${ }^{(13)}$. In ApoE*3 Leiden mice, feeding a diet enriched in animal fat (beef tallow) and cholesterol raised maternal plasma cholesterol and increased the development atherosclerosis, independently of changes in plasma cholesterol or TAG, in the offspring ${ }^{(14)}$.

Dietary trans-fatty acids (TFA), particularly those in partially hydrogenated vegetable oil (PHVO) rich in trans 18:1 isomers, adversely affect lipoprotein concentrations ${ }^{(15)}$ and increase the risk of developing atherosclerotic $\mathrm{CVD}^{(16)}$. Ruminant-derived meat and dairy products represent another source of TFA. However, while PHVO contains a wide range of isomers, the specific composition of which depends on the parent oil, ruminant-derived products contain predominantly vaccenic acid (VA, 18:1t11), which is produced by the bacterial population of the rumen. This is particularly significant as a proportion of dietary VA is converted to the conjugated linoleic acid (CLA) isomer, 18:2c9,t11, through the action of stearoyl CoA desaturase in the tissues of animals, including humans ${ }^{(17,18)}$. We have previously shown potentially beneficial

Abbreviations: HF, high fat; PHVO, partially hydrogenated vegetable oil; TFA, trans-fatty acids.

* Corresponding author: Professor A. M. Salter, fax +44 1159516 122, email Andrew.salter@nottingham.ac.uk 
effects of butter enriched in VA and $18: 2 c 9, t 11$ CLA on lipoprotein profiles ${ }^{(19)}$

In humans and animals TFA in the maternal diet can be transferred across the placenta into the circulation and tissues of the developing offspring with possible metabolic consequences $^{(20)}$. A recent cohort study suggested maternal TFA consumption, during the second trimester of pregnancy, was positively associated with fetal growth rates ${ }^{(21)}$, though studies in mice exposed to TFA-enriched milk fat show retarded growth rates $^{(22)}$. In the present study, we investigated the impact of dietary these two types of TFA during pregnancy, or pregnancy and lactation, on the development of atherosclerosis in the offspring using the ApoE*3 Leiden mouse model. We hypothesised that the impact of maternal consumption of these TFA sources would be different as a result of the differing 18:1t isomer composition of the two fats and the conversion of VA (in ruminant-derived TFA) to $18: 2 c 9, t 11$ CLA.

\section{Methods \\ Animal protocols}

All animal experiments were approved by an independent Animal Care and Use Committee and were performed under UK Home Office licence in accordance with European Union specifications. The authors have read the Animals in Research: Reporting In Vivo Experiments (ARRIVE) guidelines ${ }^{(23)}$. The animal model chosen for this study was the transgenic ApoE*3 Leiden mouse. This was the most appropriate mouse strain for the work as an animal was required that would (a) develop atherosclerosis (wild-type animals do not) and (b) do so in a manner and to an extent that is dependent upon dietary factors. In this strain the degree of atherosclerosis is directly proportional to the amount of cholesterol and amount and type of fat in the diet $^{(24)}$. Before the study the number of animals required was calculated using data from a previously reported study ${ }^{(9)}$, with the aim to have $90 \%$ power to detect an effect of maternal diet ( $\alpha=0.05, \beta=0 \cdot 1$ ). The nature of the study dictated that the feeding study was not performed blind, though all analysis (including atherosclerosis) was performed by animal number and without reference to treatment group. Animals were housed and maintained as previously described ${ }^{(9)}$. Female C57BL/6J were obtained from Harlan UK and initially maintained on standard rodent chow (Rat and Mouse Diet Number 1 Maintenance Diet (Special Diet Services)) for at least 2 weeks. According to the manufacturer, this diet contained $2.7 \%$ crude oil of which the major fatty acids were: oleic acid (0.77\%), linoleic acid $(0.69 \%)$ and palmitic acid $(0.31 \%)$. At reproductive age (approximately 7 weeks), mice were transferred to the respective diets (see below) and mated with male, heterozygous, ApoE*3 Leiden transgenic mice on a C57BL/6J background. Transgenic mice were bred in our own facility from original founder males kindly provided by Dr Louis Havekes (TNO Pharma). Heterozygous animals were used as the ApoE*3Leiden transgene is lethal in homozygotes. Pregnant females were maintained on experimental diets throughout pregnancy and, for the groups indicated, lactation. Dams and offspring were then left undisturbed until weaning at approximately $28 \mathrm{~d}$ of age. At weaning
Table 1. Fatty acid composition of diets

\begin{tabular}{|c|c|c|c|c|}
\hline Fatty acids ( $g / 100 \mathrm{~g} \mathrm{FA})$ & Diet $P$ & Diet $\mathrm{R}$ & Diet W & Diet $A$ \\
\hline $4: 0-10: 0$ & ND & $2 \cdot 3$ & ND & ND \\
\hline $12: 0$ & 0.9 & 1.6 & ND & ND \\
\hline $14: 0$ & 0.9 & $7 \cdot 0$ & $2 \cdot 4$ & ND \\
\hline $16: 0$ & $27 \cdot 1$ & $24 \cdot 0$ & $28 \cdot 0$ & $22 \cdot 8$ \\
\hline $17: 0$ & ND & 0.72 & $1 \cdot 1$ & 0.4 \\
\hline $18: 0$ & $12 \cdot 7$ & $5 \cdot 6$ & 14.4 & $29 \cdot 3$ \\
\hline $16: 1 c 9$ & ND & 1.4 & $2 \cdot 2$ & ND \\
\hline Total 18:1 c & 24.9 & $15 \cdot 6$ & $27 \cdot 4$ & $29 \cdot 6$ \\
\hline Total 18: $1 t$ & $15 \cdot 8$ & $15 \cdot 0$ & $2 \cdot 2$ & N.D. \\
\hline $18: 2 c 9, c 12$ & $10 \cdot 2$ & 11.5 & 11.9 & $10 \cdot 5$ \\
\hline $18: 3 c 9, c 12, c 15$ & 1.0 & $1 \cdot 3$ & $1 \cdot 2$ & 1.0 \\
\hline $18: 2 c 9, t 11$ & ND & 2.9 & 0.2 & ND \\
\hline
\end{tabular}

$\mathrm{ND}$, not detected $(<0.1 \%$ total fatty acid methyl esters); diet $\mathrm{P}$, enriched hydrogenated vegetable oil; diet $R$, enriched in bovine milk fat; diet $W$, enriched in a range of fats designed to approximate the fatty acid composition of a 'Western' diet; diet A, an atherogenic diet containing $15 \%$ cocoa butter and $0.25 \%$ cholesterol.

dams were killed, weighed, and blood and perirenal adipose tissue collected. Offspring were genotyped $^{(9)}$ and sexed, and female heterozygous ApoE*3 Leiden mice were transferred to the atherogenic diet (see below). The studies were restricted to females as previous work has shown that the females of this transgenic strain are considerably more susceptible to atherosclerosis than males ${ }^{(9)}$. After 3 months of ad libitum feeding, animals were killed, weighed and blood and tissues (perirenal and gonadal adipose tissue, liver and heart (including aortic root)) were harvested as previously described ${ }^{(9)}$.

\section{Experimental diets}

High-fat (HF) diets fed to pregnant/lactating females were prepared by blending powdered rodent chow (C) with $15 \%$ test fat. TFA-enriched fats were either a blend of PHVO (P) or a TFA-enriched bovine milk fat (R); the production of which has previously been described in detail ${ }^{(25)}$. In addition, further groups of females were fed on a diet containing $15 \%$ of a blend of fats $(12.5 \%$ beef tallow, $1.5 \%$ tripalmitin and $1 \%$ maize oil) designed to reflect the most extreme intakes of SFA in the Western diet $(\mathrm{W})^{(26)}$. The fatty acid composition of the diets is shown in Table 1 and the $18: 1 t$ isomer distribution of the two TFA-rich fats is shown in Fig. 1(A) (redrawn from data presented by Chardigny et al. $\left.{ }^{(25)}\right)$. While the TFA content of the $\mathrm{W}$ diet is comparable with that consumed in some human diets, the amount in the $\mathrm{P}$ and $\mathrm{R}$ diets has been deliberately elevated to elicit any specific effects. In total, seven dietary regimens were used in pregnant/lactating females: CC, PC, PP, RC, RR, $\mathrm{WC}, \mathrm{WW}$, where the first and second letters represent diet in pregnancy and lactation, respectively. Offspring were transferred onto an atherogenic diet (A) containing 15\% cocoa butter and $0.25 \%$ added cholesterol as described previously ${ }^{(9)}$. Fatty acid composition of each diet (and mouse adipose tissue) was determined by GLC essentially as described by Kraft et al. ${ }^{(27)}$.

\section{Measurement of plasma and hepatic lipids}

Plasma total and HDL-cholesterol and total TAG were determined using a Randox Autoanalyser (County Antrim) according 
to manufacturer's instructions. Non-HDL-cholesterol was calculated as the difference between total and HDL-cholesterol. Following extraction with hexane-isopropanol $(3: 2)$ hepatic TAG was determined enzymatically, as previously described ${ }^{(18)}$.

\section{Histological analyses of aortic atherosclerosis}

The heart and aortic root were frozen, stored and sectioned as previously described $^{(9)}$. From the point at which all three aortic valves could be seen intact, every third $(7 \mu \mathrm{m})$ section (moving towards the heart) was collected and stained with Oil Red O. A total of ten sections per animal were imaged and the extent of atherosclerosis quantified as previously reported ${ }^{(9)}$. In most animals, there was clearly an increase in atherosclerosis moving towards the heart, therefore section number was included in statistical analysis (see below).

\section{Statistical analysis}

For most variables analysis was performed using SPSS (version 20; IBM) and an initial comparison between a chow diet during pregnancy and lactation (CC) and test fats during pregnancy (PC, RC, WC) was performed by one-way ANOVA followed by a Dunnett post hoc test comparing the test diets against CC. All data were tested for homogeneity of variance using a Levene's test and data found not to be normally distributed were $\log ^{10}$-transformed (for which normal distribution was confirmed). The effect of changing the diet during lactation was analysed by two-way ANOVA with pregnancy diet $(\mathrm{P}, \mathrm{R}, \mathrm{W})$ and lactation diet $(\mathrm{C}, \mathrm{HF})$ as factors. Data are reported as means and standard deviations and $P$ values $<0.05$ were considered significant. Studies of developmental programming require special caution with regard to statistical design as exposure of one pregnant mouse to a particular diet will generate multiple offspring for subsequent analysis. Maternal diet was the fixed factor and the results adjusted for within litter effects $^{(28)}$. This adjustment removed the influence of having littermates within some of the groups and is an analytical approach we have used in our previous studies of $\operatorname{programming}^{(7,9)}$.

Atherosclerotic lesion area was found to be not normally distributed and still remain so when $\log ^{10}$-transformed. As a result, statistical analysis was performed on square-root transformed data (normal distribution confirmed) and data are presented as the square root of the area of staining $\left(\operatorname{sqrt} \mu \mathrm{m}^{2}\right)$. The extent of atherosclerosis clearly differed along the length of aorta observed, with the area affected increasing as sections were taken closer to the heart. To account for this, data were analysed by repeated-measures ANOVA (with section number as the repeated measure) using Genstat (version 15; VSN International). Data were also blocked for animal so that sections coming from the same animal were accounted for and for the litter from which the animal was derived. An initial analysis was performed comparing each of the pregnancy diets (including the group fed $\mathrm{C}$ diet). Further two-way ANOVA was performed with pregnancy and lactation diet as the factors.

\section{Results}

The fatty acid composition of perirenal adipose tissue in dams (culled after weaning) fed the different diets is shown in Fig. 1(B). As expected, those mothers fed the P and $\mathrm{R}$ diets throughout pregnancy and lactation accumulated TFA in their adipose tissue and the isomer distribution mirrored that of the diet. As such, animals fed the P-diet accumulated predominately 18:1t9 and those fed the R-diet accumulated predominantly $18: 1 t 11$. However, it was also notable that the R-fed animals accumulated $18: 2 c 9, t 11$, in amounts that were disproportionate to the amount in the diet. As would be expected, when dams were transferred to chow during lactation marked reductions in all TFA were observed in adipose tissue (data not shown).

There was no difference in body weight between dams fed CC throughout pregnancy and lactation and those fed any of the HF diets during pregnancy (Table 2). However, continuing HF in the diet of dams during lactation reduced maternal body weight by $11 \%(P<0 \cdot 001)$, independently of the nature of the test fat. Liver weights were also lower in dams fed HF diets during pregnancy and lactation compared with pregnancy alone (independent of type of test fat). Maternal plasma total, non-HDL- and HDL-cholesterol were all increased in animals fed either the P or R (but not W) diets compared with those fed $\mathrm{C}$ during pregnancy (Table 3). Continuing HF diets during lactation further increased total, non-HDL- and HDL-cholesterol independently of the type of test fat. Overall, the highest
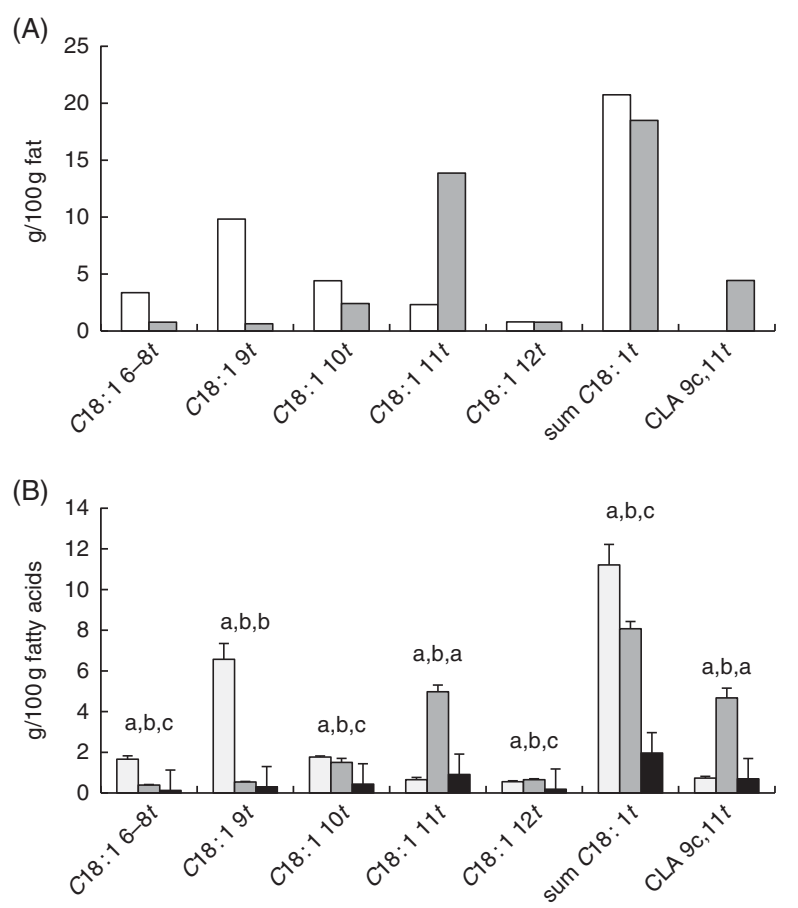

Fig. 1. Isomer distribution of $(A)$ dietary test fats and $(B)$ maternal perirenal adipose tissue. (A) The 18:1t isomer distribution of the dietary test fats is redrawn from data presented by Chardigny et al. ${ }^{(23)}$. (B) The $18: 1 t$ isomer profile of maternal perirenal adipose tissue was determined in five animals per group by GLC. ${ }^{a, b, c}$ For each isomer bars with unlike letters are significantly different $(P<0.05)$. CLA, conjugated linoleic acid; $P$, enriched hydrogenated vegetable oil $(\square)$; R, enriched in bovine milk fat $(\square)$; PP, partially hydrogenated vegetable in pregnancy and lactation $(\square)$; RR, trans fatty acid-enriched milk fat in pregnancy and lactation $(\square)$; WW, 'Western' fat in pregnancy and lactation ( $\square$ ). 
Table 2. Impact of diet on post-weaning maternal body and tissue weights $†$ (Mean values and standard deviations)

\begin{tabular}{|c|c|c|c|c|c|c|c|c|c|c|}
\hline & \multirow[b]{3}{*}{ Pregnancy diet } & \multicolumn{4}{|c|}{ Lactation diet } & & & & & \\
\hline & & \multicolumn{2}{|c|}{ C } & \multicolumn{2}{|c|}{$\mathrm{HF}$} & \multicolumn{2}{|c|}{ Total } & \multicolumn{3}{|c|}{ Two-way ANOVA $(P)$} \\
\hline & & Mean & SD & Mean & SD & Mean & SD & Pregnancy & Lactation & Pregnancy $\times$ lactation \\
\hline \multirow[t]{5}{*}{ Body weight (g) } & C & 23.1 & $2 \cdot 6$ & & & & & $0.016^{*}$ & $<0.001$ & 0.084 \\
\hline & $\mathrm{P}$ & 21.9 & 2.8 & 20.6 & 2.9 & $21 \cdot 1$ & 2.8 & & & \\
\hline & $\mathrm{R}$ & $22 \cdot 8$ & 1.6 & 20.4 & 1.7 & $21 \cdot 1$ & 2.0 & & & \\
\hline & W & $25 \cdot 7$ & 0.8 & 210 & 1.4 & 22.4 & 2.5 & & & \\
\hline & Total & $23 \cdot 3$ & 2.5 & $20 \cdot 7$ & $2 \cdot 0$ & & & & & \\
\hline \multirow[t]{5}{*}{ Liver weight (g) } & C & 1.76 & 0.36 & & & & & 0.232 & $<0.001$ & 0.259 \\
\hline & $P$ & 1.70 & 0.33 & 1.46 & 0.25 & 1.55 & 0.30 & & & \\
\hline & $\mathrm{R}$ & 1.93 & 0.26 & 1.50 & 0.31 & 1.63 & 0.35 & & & \\
\hline & W & 1.85 & 0.18 & 1.33 & 0.15 & 1.50 & 0.30 & & & \\
\hline & Total & 1.82 & 0.27 & 1.44 & 0.26 & & & & & \\
\hline \multirow{5}{*}{ Gonadal fat $(\mathrm{g})$} & C & 0.24 & 0.09 & & & & & 0.355 & 0.750 & 0.965 \\
\hline & $\mathrm{P}$ & 0.26 & 0.15 & 0.26 & 0.15 & 0.26 & 0.15 & & & \\
\hline & $\mathrm{R}$ & 0.33 & 0.16 & 0.35 & 0.19 & 0.34 & 0.18 & & & \\
\hline & W & 0.30 & 0.13 & 0.33 & 0.16 & 0.32 & 0.15 & & & \\
\hline & Total & 0.29 & 0.14 & 0.31 & 0.17 & & & & & \\
\hline \multirow{5}{*}{ Perirenal fat $(\mathrm{g})$} & C & 0.07 & 0.03 & & & & & 0.693 & 0.997 & 0.673 \\
\hline & $P$ & 0.10 & 0.07 & 0.08 & 0.07 & 0.09 & 0.07 & & & \\
\hline & $\mathrm{R}$ & 0.11 & 0.07 & $0 \cdot 10$ & 0.06 & 0.11 & 0.06 & & & \\
\hline & W & 0.10 & 0.06 & 0.12 & 0.11 & 0.11 & 0.09 & & & \\
\hline & Total & 0.10 & 0.06 & $0 \cdot 10$ & 0.08 & & & & & \\
\hline
\end{tabular}

C, chow with $15 \%$ test fat; $\mathrm{HF}$, high fat; $\mathrm{P}$, enriched hydrogenated vegetable oil; $\mathrm{R}$, enriched in bovine milk fat; $\mathrm{W}$, enriched in a range of fats designed to approximate the fatty acid composition of a 'Western' diet.

* Post hoc test not significant ( $\mathrm{P}$ v. W, $P=0.066 / \mathrm{R} v$ v. W, $P=0.061)$.

† Number of animals per group (pregnant/lactating females, respectively): $C C=13, P C=7, R C=6, W C=6, P P=11, R R=14, W W=12$.

Table 3. Impact of diet on post-weaning maternal plasma lipids $\dagger$

(Mean values and standard deviations)

\begin{tabular}{|c|c|c|c|c|c|c|c|c|c|c|}
\hline & \multirow[b]{3}{*}{ Pregnancy diet } & \multicolumn{4}{|c|}{ Lactation diet } & & & & & \\
\hline & & \multicolumn{2}{|c|}{ C } & \multicolumn{2}{|c|}{$\mathrm{HF}$} & \multicolumn{2}{|c|}{ Total } & \multicolumn{3}{|c|}{ Two-way ANOVA $(P)$} \\
\hline & & Mean & SD & Mean & SD & Mean & SD & Pregnancy & Lactation & Pregnancy $\times$ lactation \\
\hline \multirow[t]{5}{*}{ Total cholesterol $(\mathrm{mmol} / \mathrm{l})$} & C & 3.08 & 0.57 & - & & & & 0.017 & $<0.001$ & 0.089 \\
\hline & $P$ & $4.17^{\star}$ & 1.06 & 4.57 & 0.81 & $4.42^{\mathrm{a}}$ & 0.91 & & & \\
\hline & $\mathrm{R}$ & $4.44^{*}$ & 0.93 & $6 \cdot 39$ & 1.61 & $5.81^{\mathrm{b}}$ & 1.69 & & & \\
\hline & W & 3.54 & 0.76 & $5 \cdot 41$ & 0.91 & $4.79^{\mathrm{a}}$ & 1.23 & & & \\
\hline & Total & 4.06 & 0.95 & 5.54 & 1.40 & & & & & \\
\hline \multirow[t]{5}{*}{ Non-HDL-cholesterol $(\mathrm{mmol} / \mathrm{l})$} & C & 1.58 & 0.27 & & & & & 0.079 & 0.009 & 0.082 \\
\hline & $\mathrm{P}$ & $2 \cdot 24^{*}$ & 0.77 & $2 \cdot 13$ & 0.52 & $2 \cdot 17$ & 0.61 & & & \\
\hline & $\mathrm{R}$ & $2.36^{*}$ & 0.67 & 3.24 & 1.07 & 2.97 & 1.04 & & & \\
\hline & W & 1.89 & 0.43 & 2.92 & 0.76 & 2.58 & 0.82 & & & \\
\hline & Total & $2 \cdot 17$ & 0.64 & $2 \cdot 81$ & 0.94 & & & & & \\
\hline \multirow[t]{5}{*}{ HDL-cholesterol $(\mathrm{mmol} / \mathrm{l})$} & C & 1.50 & 0.37 & & & & & 0.013 & $<0.001$ & 0.397 \\
\hline & $\mathrm{P}$ & $1.93^{*}$ & 0.39 & $2 \cdot 50$ & 0.62 & $2 \cdot 29^{a}$ & 0.60 & & & \\
\hline & $\mathrm{R}$ & $2.08^{*}$ & 0.54 & $3 \cdot 16$ & 0.67 & $2.84^{\mathrm{b}}$ & 0.80 & & & \\
\hline & W & 1.65 & 0.44 & 2.49 & 0.36 & $2 \cdot 21^{\mathrm{a}}$ & 0.55 & & & \\
\hline & Total & 1.89 & 0.47 & $2 \cdot 74$ & 0.64 & & & & & \\
\hline \multirow[t]{5}{*}{ Non-HDL:HDL ratio } & C & 1.08 & 0.25 & & & & & 0.364 & 0.151 & 0.573 \\
\hline & $\mathrm{P}$ & $1 \cdot 15$ & 0.31 & 0.93 & 0.33 & 1.12 & 0.33 & & & \\
\hline & $\mathrm{R}$ & 1.17 & 0.36 & 1.03 & 0.24 & 1.07 & 0.28 & & & \\
\hline & W & $1 \cdot 19$ & 0.29 & $1 \cdot 19$ & 0.30 & 1.19 & 0.29 & & & \\
\hline & Total & 1.17 & 0.30 & 1.05 & 0.30 & & & & & \\
\hline \multirow[t]{5}{*}{ Total TAG (mmol/l) } & C & 1.01 & 0.35 & 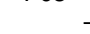 & & & & 0.220 & 0.392 & 0.572 \\
\hline & $\mathrm{P}$ & 1.44 & 0.54 & 1.12 & 0.40 & 1.24 & 0.47 & & & \\
\hline & $\mathrm{R}$ & 1.44 & 0.50 & 1.37 & 0.65 & 1.39 & 0.60 & & & \\
\hline & W & 1.09 & 0.34 & 1.11 & 0.33 & 1.11 & 0.32 & & & \\
\hline & Total & 1.33 & 0.47 & 1.22 & 0.50 & & & & & \\
\hline
\end{tabular}

$\mathrm{C}$, chow with $15 \%$ test fat; $\mathrm{HF}$, high fat; $\mathrm{P}$, enriched hydrogenated vegetable oil; $\mathrm{R}$, enriched in bovine milk fat; $\mathrm{W}$, enriched in a range of fats designed to approximate the fatty acid composition of a 'Western' diet.

a,b Mean values within a column with unlike superscript letters are significantly different.

* Significantly different from dams fed $\mathrm{C}$ during pregnancy.

† Number of animals per group (pregnant/lactating females, respectively): $C C=13, P C=7, R C=6, W C=6, P P=11, R R=14, W W=12$. 
Table 4. Impact of maternal diet on body and tissue weights of offspring* (Mean values and standard deviations)

\begin{tabular}{|c|c|c|c|c|c|c|c|c|c|c|}
\hline & \multirow[b]{3}{*}{ Pregnancy diet } & \multicolumn{4}{|c|}{ Lactation diet } & & & & & \\
\hline & & \multicolumn{2}{|c|}{ C } & \multicolumn{2}{|c|}{$\mathrm{HF}$} & \multicolumn{2}{|c|}{ Total } & \multicolumn{3}{|c|}{ Two-way ANOVA $(P)$} \\
\hline & & Mean & $\mathrm{SD}$ & Mean & SD & Mean & SD & Pregnancy & Lactation & Pregnancy $\times$ lactation \\
\hline \multirow[t]{5}{*}{ Body weight (g) } & C & $21 \cdot 0$ & $1 \cdot 2$ & & & & & 0.923 & 0.230 & 0.518 \\
\hline & $\mathrm{P}$ & $19 \cdot 6$ & 1.5 & $19 \cdot 8$ & 0.9 & $19 \cdot 7$ & 1.2 & & & \\
\hline & $\mathrm{R}$ & $20 \cdot 1$ & 1.6 & $19 \cdot 2$ & $1 \cdot 1$ & $19 \cdot 6$ & 1.4 & & & \\
\hline & W & 19.8 & 1.1 & $19 \cdot 3$ & 1.6 & 19.5 & 1.4 & & & \\
\hline & Total & 19.8 & 1.4 & $19 \cdot 4$ & $1 \cdot 2$ & & & & & \\
\hline \multirow[t]{5}{*}{ Liver weight (g) } & C & 1.27 & 0.18 & & & & & 0.505 & $<0.001$ & 0.861 \\
\hline & $\mathrm{P}$ & $1 \cdot 18$ & 0.15 & 1.09 & 0.08 & $1 \cdot 13$ & 0.12 & & & \\
\hline & $\mathrm{R}$ & $1 \cdot 17$ & 0.11 & 1.09 & 0.07 & 1.13 & 0.10 & & & \\
\hline & W & 1.14 & 0.07 & 1.07 & 0.09 & $1 \cdot 10$ & 0.09 & & & \\
\hline & Total & $1 \cdot 16$ & 0.11 & 1.08 & 0.08 & & & & & \\
\hline \multirow[t]{5}{*}{ Gonadal fat $(\mathrm{g}) \dagger$} & C & 0.20 & 0.06 & & & & & 0.009 & 0.164 & 0.548 \\
\hline & $\mathrm{P}$ & 0.18 & 0.03 & 0.14 & 0.02 & $0.16^{a}$ & 0.04 & & & \\
\hline & $\mathrm{R}$ & 0.23 & 0.11 & 0.22 & 0.11 & $0.22^{\mathrm{b}}$ & 0.11 & & & \\
\hline & W & 0.18 & 0.07 & $0 \cdot 16$ & 0.03 & $0.17^{\mathrm{a}}$ & 0.05 & & & \\
\hline & Total & 0.20 & 0.08 & 0.18 & 0.07 & & & & & \\
\hline \multirow[t]{5}{*}{ Perirenal fat $(\mathrm{g}) \dagger$} & C & 0.08 & 0.03 & & & & & $<0.001$ & 0.798 & 0.993 \\
\hline & $P$ & 0.07 & 0.02 & 0.07 & 0.02 & $0.07^{\mathrm{a}}$ & 0.02 & & & \\
\hline & $\mathrm{R}$ & 0.12 & 0.05 & 0.11 & 0.05 & $0.11^{\mathrm{b}}$ & 0.05 & & & \\
\hline & W & 0.08 & 0.03 & 0.07 & 0.01 & $0.08^{a}$ & 0.02 & & & \\
\hline & Total & 0.09 & 0.04 & 0.09 & 0.04 & & & & & \\
\hline
\end{tabular}

$\mathrm{C}$, chow with $15 \%$ test fat; HF, high fat; $\mathrm{P}$, enriched hydrogenated vegetable oil; R, enriched in bovine milk fat; W, enriched in a range of fats designed to approximate the fatty acid composition of a 'Western' diet.

a,b Mean values within a column with unlike superscript letters are significantly different.

* Number of animals per group (pregnant/lactating females, respectively): $C C=11, P C=8, R C=10, W C=10, P P=10, R R=12, W W=12$.

$\dagger$ Not normally distributed so statistical analysis performed on log-transformed data.

maternal cholesterol levels were seen in dams fed the $\mathrm{R}$ diet throughout both pregnancy and lactation (RR), which was $88 \%$ higher than that seen in animals fed chow throughout these periods (CC). However, as both non-HDL- and HDL-cholesterol increased proportionally, there was no difference in the ratio between the two. Pregnancy/lactation diet had no impact on maternal plasma TAG.

There was no impact of maternal diet on final body weight of the offspring (Table 4). Feeding the $\mathrm{R}$ diet during pregnancy increased both perirenal and gonadal adipose tissue weights in the offspring compared with both $\mathrm{P}$ and $\mathrm{W}$ diets. Fatty acid analysis of adipose tissue from the offspring of dams fed the different diets showed no difference in overall composition between the groups and no accumulation of TFA (data not shown) by 12 weeks of age. There was no impact of maternal diet on plasma total, non-HDL- or HDL-cholesterol or total plasma TAG in the offspring (Table 5). All measures of cholesterol and TAG were elevated in the offspring, compared with that of the mothers, as a result of presence of the ApoE*3 Leiden transgene and consumption of the atherogenic diet from weaning. No significant effect of maternal diet was seen of hepatic TAG.

The effect of different maternal diets during pregnancy on development of atherosclerosis in the offspring is shown in Fig. 2. It was clear that the area of atherosclerotic lesions increased progressively in sections closest to the heart, with a strong effect of section number $(P<0.001)$. When the impact of diet and section number was analysed by repeated-measures ANOVA a significant effect of pregnancy diet was seen
( $P=0.013)$ with the two TFA diets clearly resulting in lower levels of atherosclerosis than either the $\mathrm{C}$ or $\mathrm{W}$ diet. There was no significant interaction between pregnancy diet and section number $(P=0 \cdot 510)$. The impact of continuing feeding HF diets during lactation was initially analysed by repeated-measures two-way ANOVA with pregnancy diet and lactation diet as factors. This showed a specific interaction between pregnancy and lactation diet $(P=0.039)$. To clarify the impact of each of the HF diets during pregnancy and lactation they are presented (Fig. 3(a-c)) and analysed separately. In offspring of dams fed the P-rich diets there was a strong effect of lactation diet (Fig. 3(a), $P<0.001$ ) with offspring of those from mothers fed $P$ during lactation clearly displaying more atherosclerosis than those fed C. In offspring of mothers fed the R-diet (Fig. 3(b), $P=0.596$ ) or W diet (Fig. 3(c), $P=0.901$ ) there was no effect of continuing to feed the HF diets during lactation.

\section{Discussion}

The original hypothesis for this study was that fetal and neonatal exposure to the two sources of TFA in the maternal diet would have differing effects on the development of atherosclerosis due to the differing TFA isomer profiles. Few studies have looked at the effects of TFA in the maternal diet on disease susceptibility in the offspring. As already discussed such fatty acids cross the placenta and are secreted in mother's milk proportionally to the amount in the diet ${ }^{(20)}$. The present study shows that TFA derived from PHVO and those from ruminant 
Table 5. Impact of maternal diet on plasma lipids and liver TAG in offspring* (Mean values and standard deviations)

\begin{tabular}{|c|c|c|c|c|c|c|c|c|c|c|}
\hline & \multirow[b]{3}{*}{ Pregnancy diet } & \multicolumn{4}{|c|}{ Lactation diet } & & & & & \\
\hline & & \multicolumn{2}{|c|}{ C } & \multicolumn{2}{|c|}{ HF } & \multicolumn{2}{|c|}{ Total } & \multicolumn{3}{|c|}{ Two-way ANOVA $(P)$} \\
\hline & & Mean & SD & Mean & SD & Mean & SD & Pregnancy & Lactation & Pregnancy $\times$ lactation \\
\hline \multirow[t]{5}{*}{ Total cholesterol (mmol/l) } & $\mathrm{C}$ & 9.63 & $1 \cdot 21$ & & & & & 0.062 & 0.857 & 0.971 \\
\hline & P & 9.05 & 1.41 & 8.84 & 1.02 & 8.93 & $1 \cdot 18$ & & & \\
\hline & $\mathrm{R}$ & 9.86 & 1.59 & 9.92 & 2.85 & 9.89 & $2 \cdot 31$ & & & \\
\hline & W & $10 \cdot 40$ & $2 \cdot 21$ & $10 \cdot 28$ & 1.88 & $10 \cdot 34$ & 1.98 & & & \\
\hline & Total & 9.82 & 1.81 & 9.73 & $2 \cdot 13$ & & & & & \\
\hline \multirow[t]{5}{*}{ Non-HDL-cholesterol $(\mathrm{mmol} / \mathrm{l})$} & C & 7.99 & 1.41 & & & & & 0.192 & 0.964 & 0.963 \\
\hline & $P$ & 7.22 & 1.29 & 7.02 & 1.44 & $7 \cdot 11$ & 1.34 & & & \\
\hline & $\mathrm{R}$ & 7.75 & 1.40 & 7.91 & 3.23 & 7.84 & 2.54 & & & \\
\hline & W & 8.34 & 2.53 & 8.41 & $2 \cdot 36$ & 8.38 & $2 \cdot 38$ & & & \\
\hline & Total & 7.81 & 1.85 & 7.82 & 2.49 & & & & & \\
\hline \multirow{5}{*}{ HDL-cholesterol (mmol/l) } & C & 1.64 & 0.60 & & & & & 0.816 & 0.733 & 0.560 \\
\hline & $\mathrm{P}$ & 1.83 & 0.64 & 2.02 & 0.24 & 1.93 & 0.46 & & & \\
\hline & $\mathrm{R}$ & $2 \cdot 10$ & 0.34 & 2.00 & 0.71 & 2.05 & 0.57 & & & \\
\hline & W & 2.07 & 0.58 & 1.87 & 0.63 & 1.96 & 0.60 & & & \\
\hline & Total & 2.01 & 0.52 & 1.96 & 0.58 & & & & & \\
\hline \multirow[t]{5}{*}{ Total TAG (mmol/l) } & $C$ & 2.25 & 0.50 & & & & & 0.089 & 0.725 & 0.929 \\
\hline & $P$ & 2.09 & 0.38 & $2 \cdot 12$ & 0.43 & $2 \cdot 10$ & 0.40 & & & \\
\hline & $\mathrm{R}$ & 2.53 & 0.50 & 2.52 & 0.60 & 2.52 & 0.54 & & & \\
\hline & W & 2.40 & 0.54 & 2.54 & 0.76 & 2.47 & 0.66 & & & \\
\hline & Total & $2 \cdot 36$ & 0.50 & $2 \cdot 41$ & 0.64 & & & & & \\
\hline \multirow[t]{5}{*}{ Liver TAG (mg/g liver) } & C & $36 \cdot 4$ & $12 \cdot 3$ & & & & & 0.346 & 0.118 & 0.305 \\
\hline & P & 33.4 & $10 \cdot 7$ & $36 \cdot 2$ & 6.9 & $29 \cdot 4$ & 9.2 & & & \\
\hline & $\mathrm{R}$ & 43.3 & $14 \cdot 1$ & 30.5 & 13.0 & $36 \cdot 3$ & $14 \cdot 7$ & & & \\
\hline & W & 33.8 & $12 \cdot 6$ & $35 \cdot 2$ & $17 \cdot 4$ & 34.6 & $15 \cdot 1$ & & & \\
\hline & Total & $37 \cdot 1$ & $13 \cdot 1$ & 30.9 & 13.5 & & & & & \\
\hline
\end{tabular}

C, chow with $15 \%$ test fat; HF, high fat; P, enriched hydrogenated vegetable oil; R, enriched in bovine milk fat; W, enriched in a range of fats designed to approximate the fatty acid composition of a 'Western' diet.

* Number of animals per group (pregnant/lactating females, respectively): $C C=11, P C=8, R C=10, W C=10, P P=10, R R=12, W W=12$.

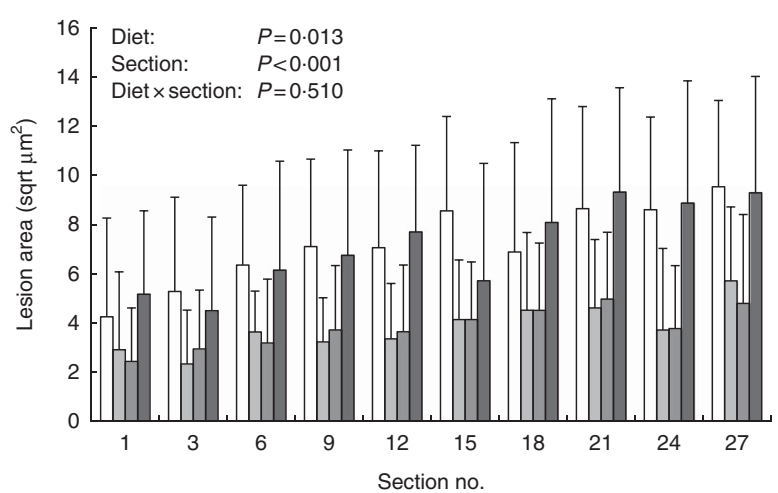

Fig. 2. Impact of maternal pregnancy diet on development of atherosclerosis in the aorta of the offspring. Atherosclerosis was determined as area of oil red-O staining of every third section $(7 \mu \mathrm{m})$ starting from where all three valve leaflets were visible. Maternal diets were CC, chow ( $\square$ ); PC, partially hydrogenated vegetable oil ( $\square$ ); RC, trans-fatty acid-enriched milk fat ( $\square$ ); and WC, a blend of fats reflecting a 'Western' diet $(\square)$. Offspring were then fed an atherogenic diet ( $15 \%$ coconut oil $+0.25 \%$ cholesterol) for 12 weeks. Data were analysed by repeated-measures (for section number) ANOVA with blocking for each individual animal and litter from which the animals were derived. $P$ values for the effect of maternal diet, section number and the interaction between the two are presented.

milk fat accumulate in the adipose tissue of dams. Those fed $\mathrm{R}$ showed a disproportionate accumulation of $18: 2 c 9, t 11$ CLA, predominantly through the action of SCD on the $18: 1 t 11$ in the dietary fat. It therefore seems appropriate to assume that the developing fetuses were exposed to different TFA isomer concentrations depending on the diet fed to the dams. As no impact of maternal diet was seen on maternal body weight, differences in susceptibility of the offspring to atherosclerosis could not be attributed to differences in maternal adiposity.

We have previously shown, using the ApoE*3 Leiden mouse model, that a low-protein maternal diet during pregnancy increased the susceptibility of the offspring to high dietary fat/ cholesterol induced atherosclerosis ${ }^{(9)}$. However, this was associated with increased plasma cholesterol concentrations in the offspring. In contrast, we recently showed that a diet enriched in cholesterol and SFA (similar to the W diet used here but including $0.25 \%$ cholesterol) also enhanced the development of atherosclerosis but in the absence of changes in plasma cholesterol in the offspring ${ }^{(14)}$. This diet resulted in maternal plasma cholesterol concentrations more than doubling through the course of pregnancy. This supports earlier studies by Napoli et al. ${ }^{(13)}$ who showed that the dramatic hypercholesterolaemia induced by feeding cholesterol to pregnant ApoE knock-out mice also increased atherosclerosis in the offspring in the absence of changes in their plasma cholesterol. In the present study, no cholesterol was added to the maternal diet, maternal cholesterol was not increased in mothers fed the W-diet, compared with those fed chow, and the offspring of these animals showed no increased susceptibility to atherosclerosis. Both the 

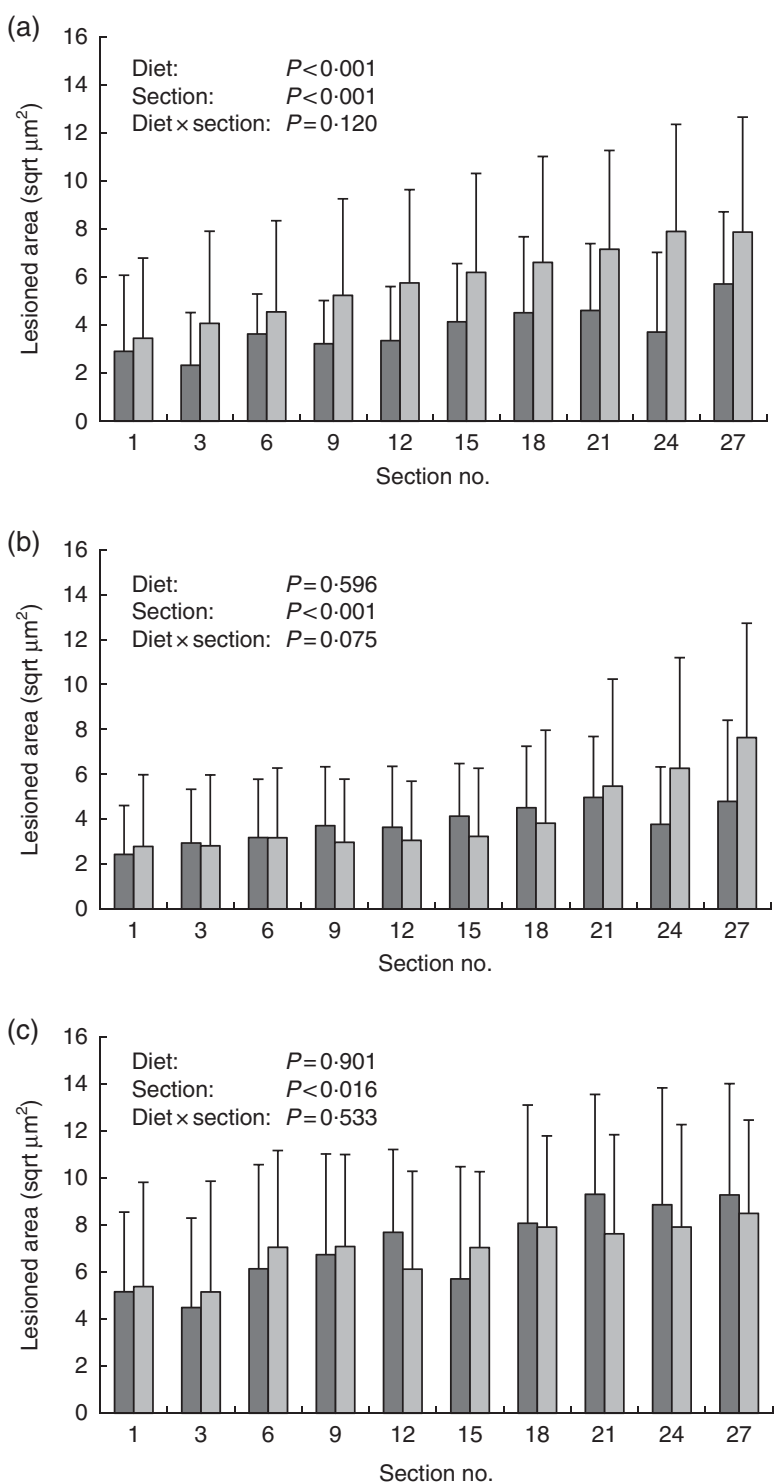

Fig. 3. Impact of high-fat maternal diets during pregnancy or pregnancy and lactation on the development of atherosclerosis in the aorta of the offspring. Atherosclerosis was determined as described in Fig. 2. Offspring were derived from mothers fed diet enriched in (a) partially hydrogenated vegetable in pregnancy (PC, $\square$ ) or pregnancy and lactation (PP, $\square$ ), (b) trans-fatty acidenriched milk fat in pregnancy (RC, $\square$ ) or pregnancy and lactation (RR, $\square$ ), (c) 'Western' fat in pregnancy (WC, $\square$ ) or pregnancy and lactation (WW, $\square$ ). Data were analysed by repeated-measures (for section number) ANOVA, with lactation diet and section number as factors, with blocking for each individual animal and litter from which the animals were derived. $P$ values for the effect of lactation diet, section number and the interaction between the two are presented.

$\mathrm{R}$ and $\mathrm{P}$ diets tended to modestly increase both non-HDL- and HDL-cholesterol with no change in the ratio of the two.

It was noticeable that the HF diets fed during pregnancy had an effect on maternal body weight and liver weight. In the absence of food intake data for the animals (as C57 mice are prone to stress-related loss of pregnancy, this data was not collected), it is difficult to pinpoint the exact reason for this. However, given that rodents effectively defend energy intake and adjust food intake when fed hyperenergetic diets ${ }^{(29)}$, it is likely that intakes of protein and micronutrients were lower in these groups. This makes comparison of the present data to our previous study of protein restriction in the apoE*3 Leiden mouse $^{(9)}$ particularly pertinent. Demands for protein and micronutrients would be particularly high during lactation.

The impact of maternal diet on the development of atherosclerosis was complex. The $\mathrm{W}$ diet showed no effect (either during pregnancy or pregnancy+lactation) compared with chow diet. However, both sources of TFA, when fed only during pregnancy, appeared to provide protection from atherosclerosis to approximately equal extents. When maternal TFA feeding was extended through lactation, much of the protective effect of $\mathrm{P}$ was lost and clear differences were seen in the distribution of atherosclerosis along the length of the aorta. Unlike our previous work investigating the impact of maternal low-protein diets, these changes in susceptibility were not associated with changes in offspring plasma cholesterol. It is possible that unidentified risk factors are responsible for the observed differences and these could include the aforementioned differences in protein and micronutrient intakes that may exist between chow-fed mice and mice fed diets of altered fatty acid composition. However, an alternative explanation may be differences in the development of lesions in neonatal life. This has been previously advocated to explain differences in the development of atherosclerosis in heterozygous apoE-deficient mice born to wild type or homozygous apoE knockout mothers ${ }^{(12)}$. While the offspring showed no differences in plasma lipids, those born of apoE-deficient mothers exhibited a greater susceptibility to atherosclerosis. The authors showed specific changes in gene expression in the aortas of affected animals and suggested that susceptibility to atherosclerosis may be imprinted during neonatal life. It is similarly possible that in our present experiment, during fetal life alterations to the biology of the developing aorta, in response to the type of fat in the mother's diet, have resulted in the differences in susceptibility to atherosclerosis in later life. In contrast, direct exposure to $\mathrm{P}$ during suckling increased susceptibility to atherosclerosis. These results suggest that the impacts of TFA on the early genesis of atherosclerosis are complex and that the effects in utero are different to the effects during suckling. The impact of TFA on atherosclerosis in adult animals are welldocumented. Bassett et al. ${ }^{(30)}$ reported that while PHVO induce atherosclerosis in LDL receptor deficient mice, ruminantderived TFA have an anti-atherogenic effect ${ }^{(31)}$, but this is the first report of their effects during early development. It might be speculated that the differential effects of TFA sources during pregnancy and lactation could be due to differences in the pattern of expression of pro- (and/or anti-)inflammatory factors and further work would be required to confirm this. It is of note, however, that such programming persists even when offspring have been challenged with an atherogenic diet for 12 weeks. This adds weight to the hypothesis that the TFA impact upon early lesion development during fetal and neonatal life. Further investigation will be required to examine early lesions in fetal and neonatal vessels in order to confirm this. Combining such an investigation with transcriptomic analysis of the fetal vasculature and maternal liver will contribute to a more detailed mechanistic understanding of the relationship between TFA, maternal cholesterol metabolism and early lesion development. 
The only other phenotypic effect of diet was an increase in adipose tissue weight, both perirenal and gonadal, in the offspring of mothers fed $\mathrm{R}$. The study provides no obvious explanation for the 'programming' of increased adiposity associated with maternal consumption of R. It should be noted that the effect was very modest (combined increased in weight of both depots equal to about $0.4 \%$ of total body weight) and that this needs to be confirmed in more detailed experiments of body composition and include measurements of food intake. Feeding HF diets during lactation had no impact upon offspring body weight or adiposity, which is consistent with some other but not all rodent studies of overfeeding in lactation ${ }^{(32)}$. However, any effect of maternal diet during suckling may have been masked by the feeding of the atherogenic diet post-weaning. It has previously been shown in rats that phenotypes programmed in lactation can be modulated by post-weaning $\operatorname{diet}^{(32,33)}$

In conclusion, consumption of TFA during pregnancy appeared to protect offspring from later atherosclerosis, independently of the isomeric distribution of the TFA. However, if feeding is maintained during lactation, this protection may be partly lost, particularly when TFA derived from $\mathrm{P}$ are consumed. The mechanisms underlying these changes remain to be established, but they are not associated with changes in lipoprotein concentrations at the point when atherosclerosis was measured.

\section{Acknowledgements}

The authors thank L. Havekes of TNO Pharma for supplying the original breeding stock of apoE*3 Leiden mice and permission to carry out the study. The authors acknowledge the support of R. Plant and C. Armett in the maintenance of the apoE*3 Leiden colony and Jim Craigon for his help with statistical analysis.

This study was supported by National Research Initiative grant no. 2008-01874 from the USDA National Institute of Food and Agriculture.

Research questions were formulated by A. M. S., S. C. L.-E., A. L. L. and J. K. Practical work was largely performed by L. G., with fatty acid analyses being undertaken in the laboratory of A. L. L. Data analysis and writing of the article was performed primarily by A. M. S. with additional support from all contributing authors.

The authors declare that there are no conflicts of interest.

\section{References}

1. Langley-Evans SC (2015) Nutrition in early life and the programming of adult disease: a review. J Hum Nutr Diet 28, Suppl. 1, 1-14.

2. Hanson MA \& Gluckman PD (2014) Early developmental conditioning of later health and disease: physiology or pathophysiology? Physiol Rev 94, 1027-1076.

3. Mone SM, Gillman MW, Miller TL, et al. (2004) Effects of environmental exposures on the cardiovascular system: prenatal period through adolescence. Pediatrics 113, Suppl. 4, 1058-1069.

4. Symonds ME, Mendez MA, Meltzer HM, et al. (2013) Early life nutritional programming of obesity: mother-child cohort studies. Ann Nutr Metab 62, 137-145.
5. Langley SC \& Jackson AA (1994) Increased systolic blood pressure in adult rats induced by fetal exposure to maternal low protein diets. Clin Sci 86, 217-222.

6. Langley-Evans SC, Welham SJ \& Jackson AA (1999) Fetal exposure to a maternal low protein diet impairs nephrogenesis and promotes hypertension in the rat. Life Sci 64, 965-974.

7. Erhuma A, Salter AM, Sculley DV, et al. (2007) Prenatal exposure to a low-protein diet programs disordered regulation of lipid metabolism in the aging rat. Am J Physiol Endocrinol Metab 292, E1702-E1714.

8. Wilson MR \& Hughes SJ (1997) The effect of maternal protein deficiency during pregnancy and lactation on glucose tolerance and pancreatic islet function in adult rat offspring. J Endocrinol 154, 177-185.

9. Yates Z, Tarling EJ, Langley-Evans SC, et al. (2009) Maternal undernutrition programmes atherosclerosis in the ApoE*3Leiden mouse. Br J Nutr 101, 1185-1194.

10. Reynolds RM, Allan KM, Raja EA, et al. (2013) Maternal obesity during pregnancy and premature mortality from cardiovascular event in adult offspring: follow-up of 1323275 person years. $\mathrm{Br}$ Med J 13, 347-f4539.

11. Samuelsson AM, Mathews PA, Argenton M, et al. (2008) Diet-induced obesity in female mice leads to offspring hyperphagia, adiposity, hypertension, and insulin resistance: a novel murine model of developmental programming. Hypertension 51, 383-392.

12. Palinski W \& Napoli C (2002) The fetal origins of atherosclerosis: maternal hypercholesterolemia, and cholesterol-lowering or antioxidant treatment during pregnancy influence in utero programming and postnatal susceptibility to atherogenesis. FASEB J 161, 348-1360.

13. Napoli C, Glass CK, Witzum JL, et al. (1999) Influence of maternal hypercholesterolaemia during pregnancy on progression of early atherosclerotic lesions in childhood: Fate of Early Lesions in Children (FELIC) study. Lancet 354, 1234-1241.

14. Tarling EJ, Ryan KPJ, Austin R, et al. (2016) Maternal high-fat feeding in pregnancy programmes atherosclerotic lesion size in the ApoE*3 Leiden mouse. Dohad J 7, 290-297.

15. Mensink RP, Zock PL, Kester ADM, et al. (2003) Effects of dietary fatty acids and carbohydrates on the ratio of serum total to HDL cholesterol and on serum lipids and apolipoproteins: a meta-analysis of 60 controlled trials. Am J Clin Nutr 77, 1146-1155.

16. Mozaffarian D \& Clarke R (2009) Quantitative effects on cardiovascular risk factors and coronary heart disease risk of replacing partially hydrogenated vegetable oils with other fats and oils. Eur J Clin Nutr 63, S22-S33.

17. Turpeinen AM, Mutanen M, Aro A, et al. (2002) Bioconversion of vaccenic acid to conjugated linoleic acid in humans. Am J Clin Nutr 76, 504-510.

18. Kuhnt K, Kraft J, Moeckel P, et al. (2006) Trans-11-18:1 is effectively delta9-desaturated compared with trans-12-18:1 in humans. BrJ Nutr 95, 752-761.

19. Lock AL, Horne CA, Bauman DE, et al. (2005) Butter naturally enriched in conjugated linoleic acid and vaccenic acid alters tissue fatty acids and improves the plasma lipoprotein profile in cholesterol-fed hamsters. J Nutr 135, 1934-1939.

20. Innis SM (2006) Trans fatty intakes during pregnancy, infancy and early childhood. Atheroscler Suppl 7, 17-20.

21. Cohen JF, Rifas-Shiman SL, Rimm EB, et al. (2011) Maternal trans fatty acid intake and fetal growth. Am J Clin Nutr 94, 1241-1247.

22. Kavanagh K, Sajadian S, Jenkins KA, et al. (2010) Neonatal and fetal exposure to trans-fatty acids retards early growth and adiposity while adversely affecting glucose in mice. Nutr Res 30, 418-426. 
23. Kilkenny C, Browne WJ, Cuthill IC, et al. (2010) Improving biscience research reporting: the ARRIVE guidelines for reporting animal research. PLOS Biol 8, e1000412.

24. Groot PH, van Vlijmen BJ, Benson GM, et al. (1995) Quantitative assessment of aortic atherosclerosis in APOE*3 Leiden transgenic mice and its relationship to serum cholesterol exposure. Arterioscler Thromb Vasc Biol 16, 926-933.

25. Chardigny JM, Destaillats F, Malpuech-Brugère C, et al. (2008) Do trans fatty acids from industrially produced sources and from natural sources have the same effect on cardiovascular disease risk factors in healthy subjects? Results of the trans Fatty Acids Collaboration (TRANSFACT) study. Am J Clin Nutr 87, 558-566.

26. Kris-Etherton P, Lefevre M, Mensink RP, et al. (2012) Trans fatty acid intakes and food sources in the US population: NHANES 1999-2002. Lipids 47, 931-940.

27. Kraft J, Hanske L, Mockel P, et al. (2006) The conversion efficiency of trans-11 and trans-12 $18: 1$ by delta 9-desaturation differs in rats. J Nutr 136, 1209-1214.
28. Festing MFW (2006) Design and statisitical methods in studies using animal models of development. ILAR J 47, 5-14.

29. Sampey BP, Vanhoose AM, Winfield HM, et al. (2011) Cafeteria diet is a robust model of human metabolic syndrome with liver and adipose inflammation: comparison to highfat diet. Obesity 19, 1109-1117.

30. Bassett CMC, McCullough RS, Edel AL, et al. (2009) trans-Fatty acids in the diet stimulate atherosclerosis. Metabolism 58, 1802-1808.

31. Bassett CMC, Edel AL, Patenaude AF, et al. (2010) Dietary vaccenic acid has antiatherogenic effects in $\mathrm{LDLr}^{-/-}$mice. J Nutr 140, 18-24.

32. Akyol A, McMullen S \& Langley-Evans SC (2012) Glucose intolerance associated with early-life exposure to maternal cafeteria feeding is dependent upon post-weaning diet. Br J Nutr 107, 964-978.

33. Gugusheff JR, Vithayathil M, Ong ZY, et al. (2013) The effects of prenatal exposure to a 'junk food' diet on offspring food preferences and fat deposition can be mitigated by improved nutrition during lactation. J Dev Orig Health Dis 4, 348-357. 